\title{
Intelligent Load Frequency Controller Using GrADP for Island Smart Grid with Electric Vehicles and Renewable Resources
}

\author{
Yufei Tang ${ }^{\mathrm{a}}$, Jun Yang ${ }^{\mathrm{b}}$, Jun Yan ${ }^{\mathrm{a}}$, Haibo $\mathrm{He}^{\mathrm{a}, *}$ \\ ${ }^{a}$ Department of Electrical, Computer and Biomedical Engineering, University of Rhode \\ Island, Kingston, RI, 02881, USA. \\ ${ }^{b}$ School of Electrical Engineering, Wuhan University, Wuhan, Hubei Province, 430072, \\ China.
}

\begin{abstract}
Increasing deployment of intermittent power generation from renewable resources in the smart grid, such as photovoltaic (PV) or wind farm, will cause large system frequency fluctuation when the load-frequency control (LFC) capacity is not enough to compensate the unbalance of generation and load demand. Even worse, the system inertia will decrease when the smart grid is in island operating mode, which would degrade system damping and cause system instability. Meanwhile, electric vehicles (EVs) will be widely used by customers in the near future, where the EV station could be treated as dispersed battery energy storage. Therefore, the vehicle-to-grid (V2G) technology can be employed to compensate for inadequate LFC capacity, thus improving the island smart grid frequency stability. In this paper, an on-line reinforcement learning $(\mathrm{RL})$ based method, called goal representation adaptive dynamic programming (GrADP), is employed to adaptive control of units in an island smart grid. In the controller design, adaptive supplementary control signals are provided to proportional-integral-derivative (PID) controller by GrADP in a real-time manner. Comparative simulation studies on a benchmark smart grid with mi-
\end{abstract}

\footnotetext{
An abbreviated version of some portions of this article appeared in [1] as part of the IJCNN 2014 Conference Proceedings, published under IEEE copyright.

* Corresponding author

Email address: he@ele.uri.edu (Haibo He)
}

Preprint submitted to Journal of Neurocomputing

March 23, 2015 
cro turbine (MT), EVs, PV array and wind power are carried out among the GrADP controller, the original PID controller and the particle swarm optimization (PSO) based fuzzy logic controller. Simulation results demonstrate competitive performance and satisfied learning ability of the GrADP based coordinate controller. Moreover, the impact of signal transmission delay on the control performance is also considered, and suggestions to address this issue are given in the paper.

Keywords: Load-frequency control (LFC), Goal representation adaptive dynamic programming (GrADP), fuzzy logic, electric vehicle (EV), signal transmission delay

\section{Introduction}

As the increasing environmental and energy security concerns, renewable energy from wind or solar as well as electric vehicles (EVs) will be widely deployed in the smart grid in the next decade. The large-scale integration of these

5 new types of generation and load in power grids will have significant impact on grid operation, planning and stability control [2]. Among all the critical issues, an important one is large system frequency fluctuation caused by intermittent renewable energy and changing load conditions in island operating smart grid, where the load-frequency control (LFC) capacity is not enough to compensate unbalance of generation and demand. Therefore, in this paper, we focus on the design of a power system frequency oscillation damping controller based on goal representation adaptive dynamic programming (GrADP). The impact of signal transmission delay between the units and the smart-grid dispatch system (SGDS) on the control performance are also considered, and suggestions to address this issue are given.

In traditional load-frequency controller design, e.g. the proportional-integral (PI) controller, a linearized power system model near the operating point is used [3]. The nominal design model is obtained for a particular operating condition. After off-line tuning of the parameters, extensive field testing is done at the time 
of commissioning. A PI controller based on this approach can be well tuned to an operating condition and provide excellent frequency damping over a certain range around the design point. However, new types of generation and load resources in the smart grid require us to be more careful on the aforementioned "linearity" assumption. In island smart grid with photovoltaics (PVs), EVs and

25 wind turbines, system state parameters and operating conditions are changing frequently, therefore, the fixed parameters of PI controllers may not be optimal for the whole set of possible operating conditions and configurations. Considering the facts above, it is desirable to develop a stabilizer which has the ability to adjust its parameters on-line according to its environment.

Meanwhile, with the improvement of vehicle-to-grid (V2G) technique, EVs can act as controllable loads and mobile storage devices, which will bring new solution for frequency regulation service [4]. By providing active power to load or absorbing extra active power from generation, EVs could quickly compensate the active power gap between generation and load, thus improving the 35 power grid frequency stability. Intensive investigations on EVs have been carried out in the community, such as load frequency control using V2G system considering the customer convenience of EVs [5], integration of $\mathrm{V} 2 \mathrm{G}$ in a real power system in western Denmark [6], fuzzy logic controller based V2G for frequency regulation [7] [8], supplementary LFC with both EVs and heat pump

40 water heaters 9], coordinated frequency control between wind power and EVs [10, supplementary load frequency control with storage battery operation considering state-of-charge (SOC) under large-scale wind power penetration [11, and among others.

Based on the aforementioned discussions and inspired by the research in 45 [12, the on-line GrADP is adopted in this paper to provide supplementary control signal to the original proportional-integral-derivative (PID) controller. The advantage of adaptive dynamic programming (ADP) based design is that no system model is required for such supplementary controller [13]. Once a system state is observed and send to the GrADP controller, adaptive supplementary 50 control signals are generated according to the utility function and then fed 
into PID controller. Moreover, the goal representation network in the adopted GrADP algorithm could provide much more informative internal reinforcement signal to the critic network, which facilitate the learning process and improve the control performance. Such GrADP design has been tested on virtual reality 55 (VR) platform with numerical balancing benchmarks [14][15], maze navigation [16], energy storage based power system damping control [17, and power system stability control for a real-size wind farm [18, demonstrating promising control performance over the traditional ones.

The rest of this paper is organized as follows. Section 2 introduces the benchmark power system used in this paper and the formulation of the problem. Section 3 presents the on-line learning GrADP algorithm, including the structure of this method and the related learning strategies. Section 4 gives the detailed system simulation methodology and load frequency controller design, including the PSO based fuzzy logic controller and the GrADP based supplementary controller. Simulation results are presented in Section 5 , and conclusions are drawn in Section 6.

\section{Benchmark Power System Description and Problem Formulation}

The benchmark power system used in this paper is shown in Fig. 1. With the same model, the smart grid can be operated in two alternative modes, i.e., island mode and grid-connected mode. If the smart grid is in grid-connected mode, the majority of the loads can be supplied by the connected main grid and the system could be controlled by distribution management system (DMS). In this paper, the benchmark power system is in island operating mode, where the system power flow are balanced by local generation and the system is controlled

75 by local smart-grid dispatch system (SGDS). Micro turbine (MT), photovoltaic (PV) arrays and wind turbine provide active power to local load, such as smart homes. Two EV stations could be treated as dispersed battery energy storages [19] to compensate the unbalance of generation and load demand. All the system states (i.e., system frequency, active power) are measured by distributed sensors 
and then transmitted to the SGDS through communication channel. These signals are processed by SGDS to generate control signals and then these control signals are sent back to each unit (i.e., MT, EV stations) for maintaining the system stability.

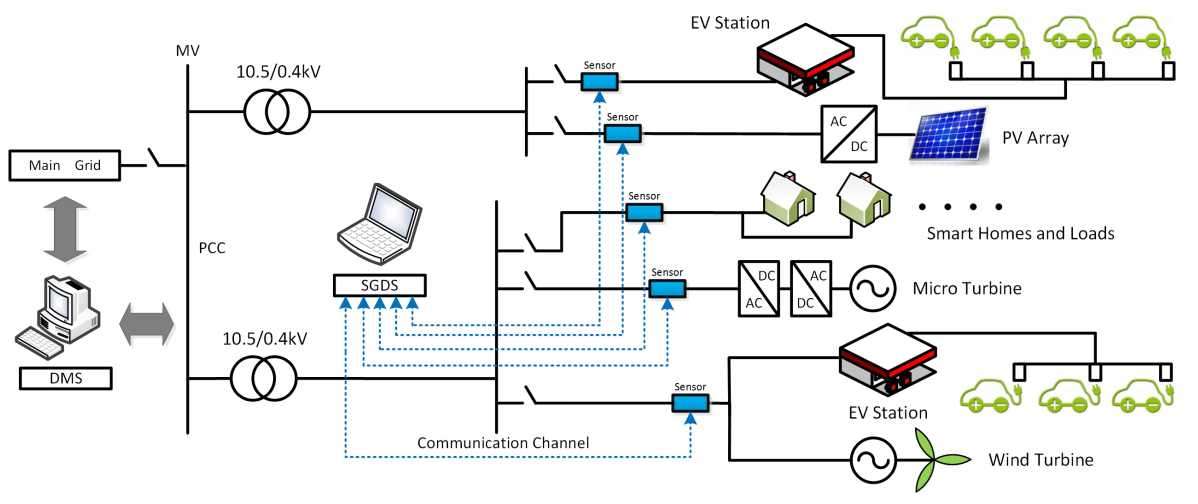

Figure 1: Island smart grid with EVs, PVs and wind turbine

Because this system is in island operating, the LFC capacity is not adequate to quickly damp the frequency oscillation. After incorporating the EVs into LFC, the system inertia could be increased, thus improving the island smart grid frequency stability. Fig. 22 shows the system frequency dynamics after active power disturbance with EVs and without EVs. It is shown that with EVs, the system frequency damping performance after the disturbance could be largely improved. We should also notice that the signal transmission in the communication channel will introduce transmission delay, which will influence the control performance. As can be observed in this figure, when the transmission delay increase to $150 \mathrm{~ms}$ [20], the system damping performance is degraded compared with $0 \mathrm{~ms}$ delay. Moreover, it is interesting to use advanced intelligent control algorithms to further improve the control performance and observe the impact of signal transmission delay on these methods. The following section will briefly introduces the adopted RL based method, followed by the controller design for this benchmark system. 


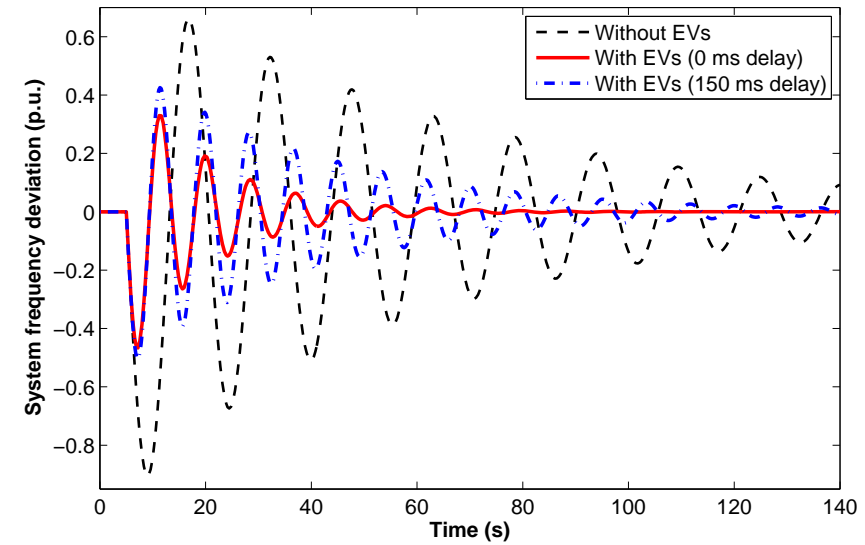

Figure 2: Island smart grid frequency response with and without EVs consider signal transmission delay

\section{On-line Reinforcement Learning Method: GrADP Algorithm}

The adopted on-line learning method, as shown in Fig. 3 is called goal representation adaptive dynamic programming (GrADP), which is a reinforcement learning algorithm from the family of adaptive critic designs (ACDs) developed in 21. It includes three function approximation networks: a goal network, a critic network, and an action network. The critic network learns to approximate the cost-to-go function in Bellman's equation; the action network learns to generate a control policy that minimizes the cost-to-go approximated by the critic network; the goal network provides an adaptive internal reinforcement signal to the critic network to improve generalization and learning capability 22]. Specifically, the cost-to-go function is defined as follows:

$$
J[x(i), i]=\sum_{t=i}^{\infty} \gamma^{t-i} U[x(t), u(t), i]
$$

where $x(t)$ is the state vector of the system, $u(t)$ is the control action, $U$ is the utility function, and $\gamma$ is a discount factor.

In this paper, all the three networks are implemented as neural networks with a three-layer architecture that includes only one hidden layer. Meanwhile, 
the learning principles can also be generalized and extended to any arbitrary function approximator.

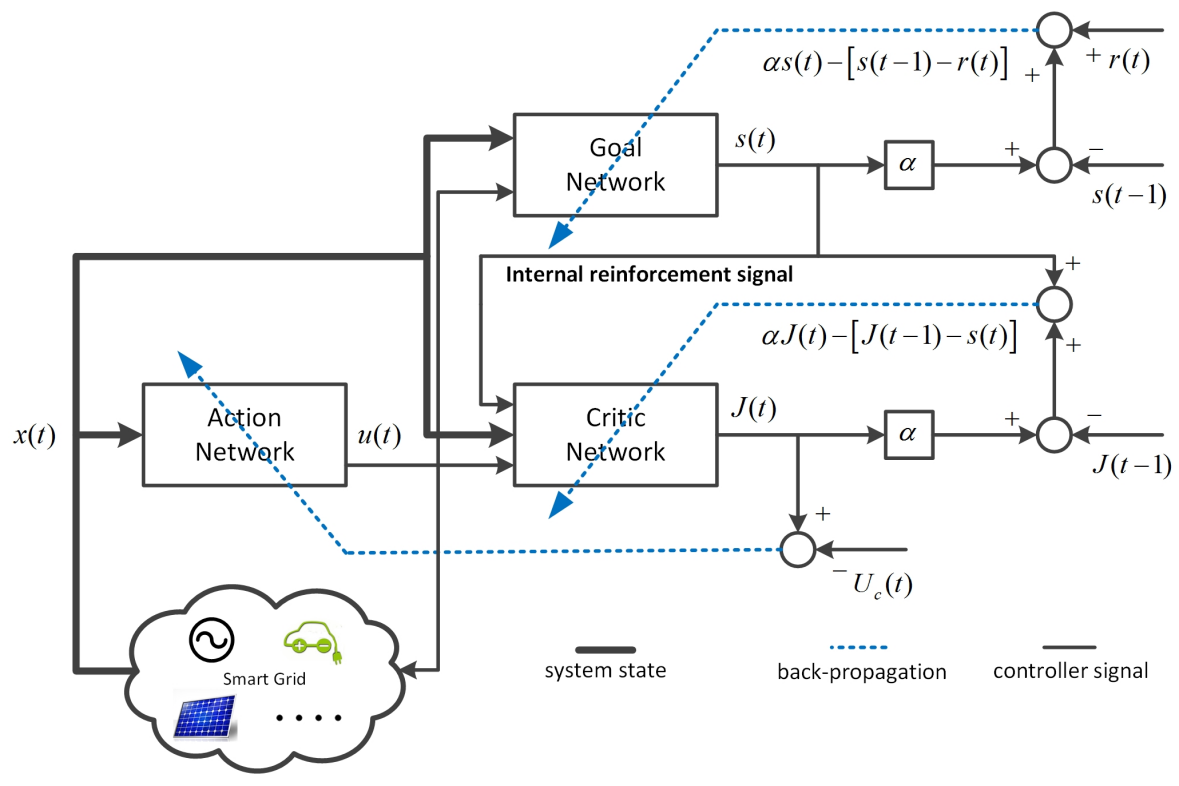

Figure 3: Structure of the adopted GrADP Algorithm [1]

\subsection{Goal Network Training}

As indicated in equation (1), the system performance cost is expressed in a compact form. The objective of dynamic programming is to choose the control sequence $u(t)$ so the cost function $J$ is minimized:

$$
J^{*}(x(t))=\min _{u(t)}\left\{U(x(t), u(t))+\gamma J^{*}(x(t+1))\right\}
$$

This is the foundation for implementing dynamic programming by working backward in time domain. Similar as in 15] 16, in this structure, $J$ can be estimated by minimizing the following error over time:

$$
\left\|E_{h}\right\|=\frac{1}{2} \sum_{t}[J(t)-r(t)-\alpha J(t+1)]
$$

When $E_{h}=0$ for all $t,(3)$ indicates:

$$
J(t)=r(t)+\alpha J(t+1)
$$


With one time step backward, we can write:

$$
J(t-1)=r(t-1)+\alpha J(t)
$$

${ }_{125}$ From equation (4) and (5), the objective function to be minimized in the goal network can be written as:

$$
\left\{\begin{array}{c}
e_{g}(t)=\alpha J(t)-[J(t-1)-r(t-1)] \\
E_{g}(t)=\frac{1}{2} e_{g}^{2}(t)
\end{array}\right.
$$

and the high-level conceptual back-propagation path is:

$$
\frac{\partial E_{g}(t)}{\partial \omega_{g}(t)}=\frac{\partial E_{g}(t)}{\partial J(t)} \frac{\partial J(t)}{\partial s(t)} \frac{\partial s(t)}{\partial \omega_{g}(t)}
$$

For the three-layer critic network, the weights of the output layer and the hidden layer are updated respectively as follows

$$
\left\{\begin{array}{c}
\Delta \omega_{g_{i}}^{(2)}=\eta_{g}(t)\left[-\frac{\partial E_{g}(t)}{\partial \omega_{g_{i}}^{(2)}(t)}\right] \\
\Delta \omega_{g_{i, j}}^{(1)}=\eta_{g}(t)\left[-\frac{\partial E_{g}(t)}{\partial \omega_{g_{i, j}}^{(1)}(t)}\right]
\end{array}\right.
$$

\subsection{Critic Network Training}

Once the $s(t)$ signal is obtained from the goal network, it will be used as an input to the critic network, and also be used to define the error function to adjust the parameters of the critic network:

$$
\left\{\begin{array}{c}
e_{c}(t)=\alpha J(t)-[J(t-1)-s(t)] \\
E_{c}(t)=\frac{1}{2} e_{c}^{2}(t)
\end{array}\right.
$$

And the back-propagation path is:

$$
\frac{\partial E_{c}(t)}{\partial \omega_{c}(t)}=\frac{\partial E_{c}(t)}{\partial J(t)} \frac{\partial J(t)}{\partial \omega_{c}(t)}
$$

135 The weights of the output layer and the hidden layer of the critic network are updated respectively as follows

$$
\left\{\begin{aligned}
\Delta \omega_{c_{i}}^{(2)} & =\eta_{c}(t)\left[-\frac{\partial E_{c}(t)}{\partial \omega_{c_{i}}^{(2)}(t)}\right] \\
\Delta \omega_{c_{i, j}}^{(1)} & =\eta_{c}(t)\left[-\frac{\partial E_{c}(t)}{\partial \omega_{c_{i, j}}^{(1)}(t)}\right]
\end{aligned}\right.
$$




\subsection{Action Network Training}

The procedure of adapting the action network is similar to the classic ADP approach to implicitly back-propagate the error between a desired ultimate object $U_{c}$ and the approximate $J$ function of the critic network [13. $U_{c}$ is in accordance with the signal of the reinforcement when the state conducted by the action implies a success. Therefore, the error function used to update the parameters of the action network is:

$$
\left\{\begin{array}{c}
e_{a}(t)=J(t)-U_{c}(t) \\
E_{a}(t)=\frac{1}{2} e_{a}^{2}(t)
\end{array}\right.
$$

where the $U_{c}$ is set to 0 to represent success, for the reason that the ultimate goal is to damp the system frequency deviation to 0 . Since the action network is connected with both goal network and critic network, the back-propagation path will consist of two parts as follows:

$$
\left\{\begin{array}{c}
\frac{\partial E_{a}(t)}{\partial \omega_{a}(t)}=P_{a, c}(t)+P_{a, g}(t) \\
P_{a, c}(t)=\frac{\partial E_{a}(t)}{\partial J(t)} \frac{\partial J(t)}{\partial u(t)} \frac{\partial u(t)}{\partial \omega_{a}(t)} \\
P_{a, g}(t)=\frac{\partial E_{a}(t)}{\partial J(t)} \frac{\partial J(t)}{\partial s(t)} \frac{\partial s(t)}{\partial u(t)} \frac{\partial u(t)}{\partial \omega_{a}(t)}
\end{array}\right.
$$

And the weights of the output layer and hidden layer in the action network are updated as follows:

$$
\left\{\begin{aligned}
\Delta \omega_{a_{i}}^{(2)} & =\eta_{a}(t)\left[-\frac{\partial E_{a}(t)}{\partial \omega_{a_{i}}^{(2)}(t)}\right] \\
\Delta \omega_{a_{i, j}}^{(1)} & =\eta_{a}(t)\left[-\frac{\partial E_{a}(t)}{\partial \omega_{a_{i, j}}^{(1)}(t)}\right]
\end{aligned}\right.
$$

\section{Design of the Load Frequency Controller}

The load frequency controller with the island benchmark power system is shown in Fig. 4. It is modeled and implemented in Matlab/Simulink environment, where the MT fuel system, turbine and two EVs are represented by transfer functions [19. The signal transmission delay is modeled by using the "transport delay" blocks in Simulink library. The parameters of the system are illustrated in Tab. 1. The active power fluctuation from the PVs, wind power 


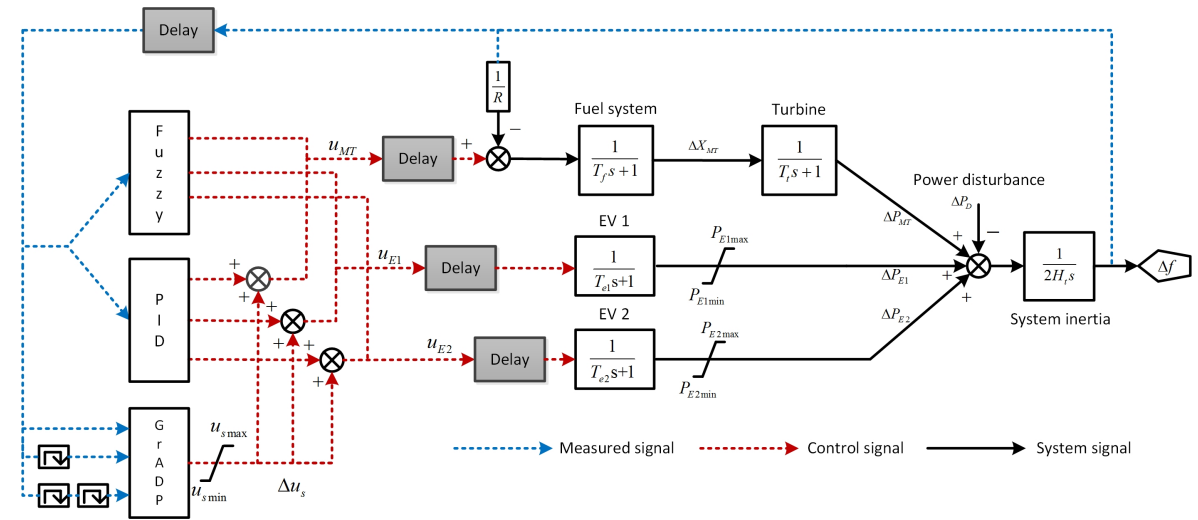

Figure 4: The configuration of the load frequency controller with island smart grid consider signal transmission delay, where a PID controller, a PSO optimized fuzzy logic controller and a GrADP based supplementary controller are illustrated

and load changing are modeled as power disturbance $\Delta P_{D}$, which will cause system frequency oscillation. The deviation of the system active power and the inertia of the island smart grid $H_{t}$ are then used to form the system frequency deviation $\Delta f$. In the original design, a PID controller is used to control the MT and two EVs to damp the frequency oscillation. In this paper, a particle swarm optimization (PSO) based fuzzy logic controller and a GrADP based supplementary controller are used to compare the damping performance.

\subsection{PSO optimized fuzzy logic controller design}

The overall structure of the PSO based fuzzy logic controller is shown in Fig. 5 The system frequency deviation $\Delta f$ and its derivative are the two input signals, and the provided control signal is used by the LFC participant units. The scaling factors for the two input signals and the one output signal are $K_{e}, K_{e c}$ and $K_{u}$, respectively. The fuzzy logic controller used in this paper is Mamdani-Type fuzzy inference system 23] 24]. First, the membership functions for the input and output variables are shown in Fig. 6. As can be observed from this figure, triangular and trapezoidal membership functions are both applied. The 7-segments membership functions are defined as negative big (NB), negative 
Table 1: Parameters of the benchmark system

\begin{tabular}{c||c||c||c}
\hline \hline Component & Parameter & Description & Value \\
\hline \hline \multirow{2}{*}{ MT } & $T_{f}$ & Fuel system time constant & $10 \mathrm{~s}$ \\
\cline { 2 - 4 } & $T_{t}$ & Turbine time constant & $0.1 \mathrm{~s}$ \\
\cline { 2 - 4 } & $\delta_{m t}$ & Power ramp rate limit & $0.01 \mathrm{MW} / \mathrm{second}$ \\
\hline \multirow{3}{*}{ EV1 } & $T_{e 1}$ & EV1 time constant & $1 \mathrm{~s}$ \\
\cline { 2 - 4 } & $P_{E 1 \text { max }}$ & Maximum energy & $+0.1 \mathrm{MWh}$ \\
\cline { 2 - 4 } & $P_{E 1 \text { min }}$ & Minimum energy & $-0.1 \mathrm{MWh}$ \\
\cline { 2 - 4 } & $T_{e 2}$ & EV2 time constant & $1 \mathrm{~s}$ \\
\cline { 2 - 4 } & $P_{E 2 \max }$ & Maximum energy & $+0.1 \mathrm{MWh}$ \\
\cline { 2 - 4 } & $P_{E 2 \min }$ & Minimum energy & $-0.1 \mathrm{MWh}$ \\
\hline \multirow{2}{*}{ Grid } & $H_{t}$ & Island Smart grid inertia & $7.11 \mathrm{~s}$ \\
\hline \hline
\end{tabular}

medium (NM), negative small (NS), zero (ZO), positive small (PS), positive medium (PM), and positive big (PB).

There are totally 49 fuzzy rules are considered in this design, which is shown in Tab. 2. This rule base works on the vectors composed of the two input signals. The "T-norms" is based on interpreting the "and" by taking the minimum of the two membership values. Crisp input signals are first mapped to linguistic values, and then combined based on all the rules by using "sum" method. Finally, for converting output to a crisp value, the "centroid" method is used for defuzzification [25].

The PSO algorithm is employed to search the optimal scaling factors in the solution space. Therefore, there are three parameters should be optimized: $K_{e}$, $K_{e c}$ and $K_{u}$. The searching range for these three parameters are [1 10000], $[1 \sim 1000]$, and $[0.01 \sim 0.1]$, respectively. The particles number, particle size, maximum velocity, initial learning rate, final learning rate, $c_{1}$, and $c_{2}$ are chosen as $10,3,10 \%$ of the searching upper limit corresponding to each parameter, 0.9, $0.2,2,2$, respectively. The fitness function design in the PSO is critical for the 


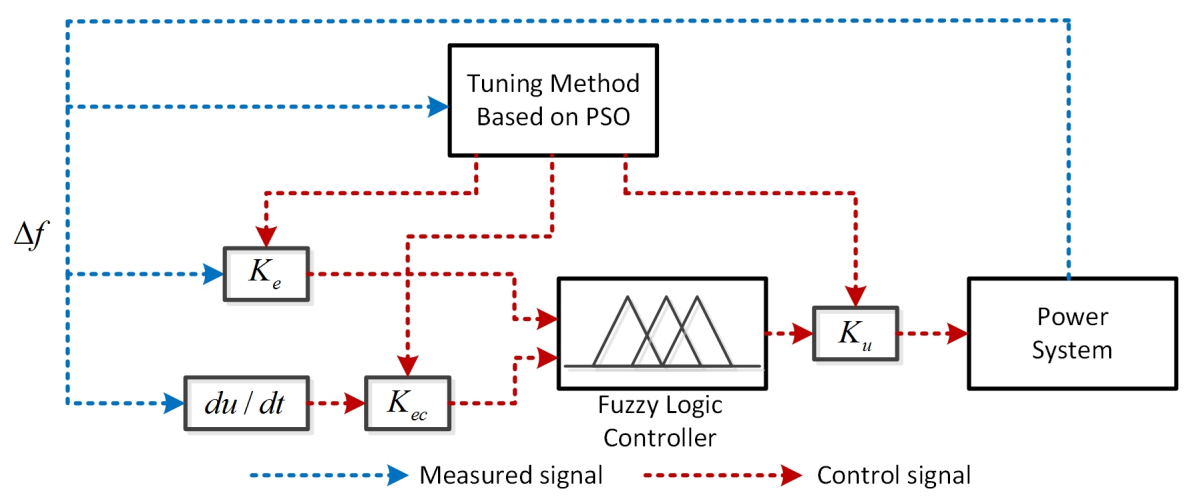

Figure 5: Structure of the PSO optimized fuzzy logic controller

\subsection{GrADP based controller design}

The structure of the GrADP algorithm has been introduced in Section 3 The input of the GrADP controller is designed as follows:

$$
x(t)=\left[\begin{array}{lll}
\Delta f(t) & \Delta f(t-1) & \Delta f(t-2)
\end{array}\right]
$$




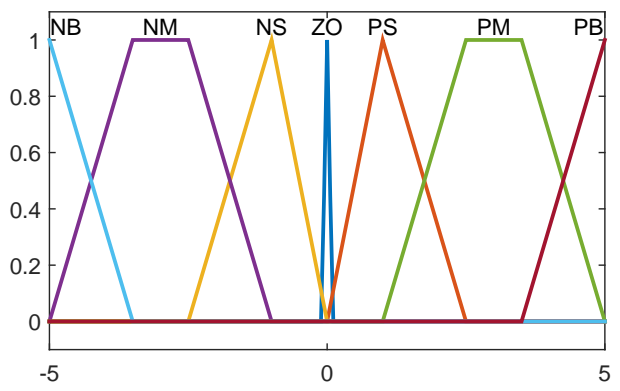

(a)

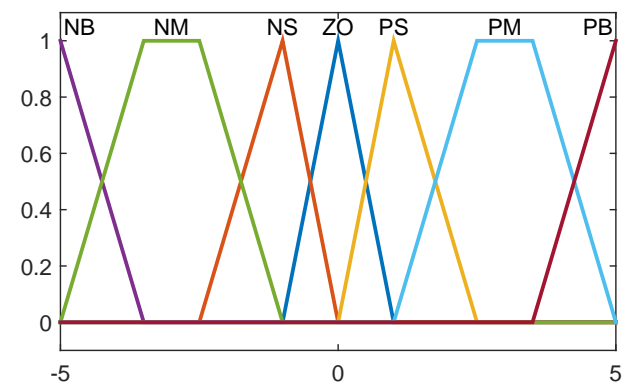

(b)

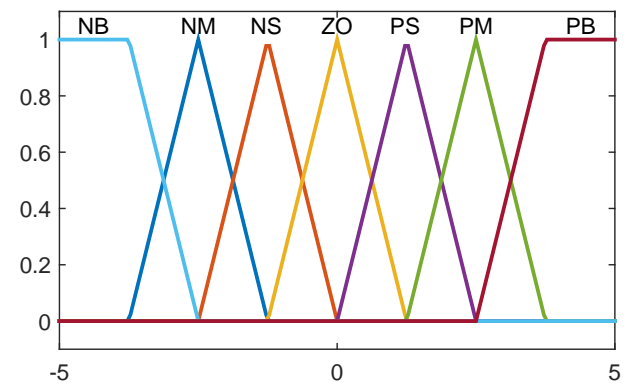

(c)

Figure 6: Fuzzy membership functions used in this paper; (a) and (b) are input patterns; (c) is output pattern 
Table 2: Fuzzy Rules Set

\begin{tabular}{|c|c|c|c|c|c|c|c|c|}
\hline \multirow{2}{*}{\multicolumn{2}{|c|}{ Inputs }} & \multicolumn{7}{|c|}{$\Delta f$} \\
\hline & & NB & NM & NS & $\mathrm{ZO}$ & PS & PM & $\mathrm{PB}$ \\
\hline \multirow{7}{*}{$\Delta f^{\prime}$} & NB & NB & NB & NB & NB & $\mathrm{NM}$ & NS & $\mathrm{ZO}$ \\
\hline & NM & NB & NB & NB & $\mathrm{NM}$ & NS & $\mathrm{ZO}$ & PS \\
\hline & NS & NB & NB & NM & NS & $\mathrm{ZO}$ & PS & $\mathrm{PM}$ \\
\hline & $\mathrm{ZO}$ & NB & NM & NS & $\mathrm{ZO}$ & PS & PM & PB \\
\hline & PS & NM & NS & $\mathrm{ZO}$ & PS & PM & PB & $\mathrm{PB}$ \\
\hline & PM & NS & $\mathrm{ZO}$ & PS & PM & $\mathrm{PB}$ & $\mathrm{PB}$ & $\mathrm{PB}$ \\
\hline & PB & $\mathrm{ZO}$ & PS & $\mathrm{PM}$ & $\mathrm{PB}$ & $\mathrm{PB}$ & PB & $\mathrm{PB}$ \\
\hline
\end{tabular}

where $\Delta f(t)$ is the measured system frequency deviation at time $t$. The previous two sampling signals are also used here to provide the controller more system dynamic information under disturbance [27, 28. The output of the GrADP controller is $\Delta u_{s}$, which will be added to the three outputs of the PID controller to form new control actions to the MT and EVs. In order to prevent the adaptive control actions to dominate the PID control, a limitation unit of \pm 0.02 is imposed to $\Delta u_{s}$. The reinforcement signal of the GrADP controller is designed as follows 29]:

$$
\left\{\begin{array}{c}
Q=\operatorname{diag}\left[\begin{array}{ccc}
1 & 0.5 & 0.5^{2}
\end{array}\right] \\
r(t)=-x(t) * Q * x(t)^{\prime}
\end{array}\right.
$$

Once the GrADP controller is initialized, it will be plugged into the system and works in the following procedure [17] [18]:

1. The action network receives the measured power system state $x(t)$ and uses it to generate the control signal $\Delta u_{s}$ to the PID controllers.

2 . The goal network uses the external reinforcement signal $r(t)$, power system state $x(t)$ and the control signal $\Delta u_{s}$ to generate the internal reinforcement signal $s(t)$.

3. Then the critic network uses the internal reinforcement signal $s(t)$, power 
system state $x(t)$, and the control signal $\Delta u_{s}$ to estimate the cost function $J$.

4. The goal network will update its weights according to equation (6), (7) and (8) until the stop criterion is satisfied.

5. The critic network will update its weights according to equation $(9), 10)$ and (11) until the stop criterion is satisfied.

6. The action network will update its weights according to equation (12), (13) and (14) until the stop criterion is satisfied.

7. Above steps (1) to (6) are repeated in each sampling time step until the end of the simulation.

In this paper, the learning procedure between the goal network and the critic network are implemented sequentially, while in [18] a cooperative learning strategy is used. Such sequential learning strategy is more easy to implement and holds the similar control performance as cooperative learning strategy. The general parameters used in the GrADP controller are shown in Tab. 3. In this table, $K_{i}$ is the input number of action network; $K_{o a}, K_{o g}$ and $K_{o c}$ are the output number of action network, goal network and critic network, respectively; $K_{h a}$, $K_{h g}$ and $K_{h c}$ are the hidden neuron number of action network, goal network and critic network, respectively. Based on the aforementioned input and reinforcement signal design, $K_{i}=3, K_{o a}=K_{o g}=K_{o c}=1$, and $K_{h a}=K_{h g}=K_{h c}=6$ are used in this paper.

Table 3: General parameters of the GrADP based load frequency controller

\begin{tabular}{c||c||c||c}
\hline \hline Network & Action Net. & Goal Net. & Critic Net. \\
\hline \hline Inputs Number & $K_{i}$ & $K_{i}+1$ & $K_{i}+2$ \\
\hline Outputs Number & $K_{o a}$ & $K_{o g}$ & $K_{o c}$ \\
\hline Hidden Neurons & $K_{h a}$ & $K_{h g}$ & $K_{h c}$ \\
\hline Activation Function & Sigmoid & Sigmoid & Sigmoid \\
\hline \hline
\end{tabular}

The specific parameters used in the GrADP controller are summarized in 
Tab. 4. In this table, $\eta_{a}(0), \eta_{g}(0)$ and $\eta_{c}(0)$ are the initial learning rate of the action network, goal network and critic network, respectively; $\eta_{a}(t)$ is the learning rate of the action network which is decreased by 0.05 every 5 time step until it reach $\eta_{a}(f)$ and stay thereafter; $\eta_{g}(t)$ is the learning rate of the goal network which is decreased by 0.05 every 5 time step until it reach $\eta_{g}(f)$ and stay thereafter; $\eta_{c}(t)$ is the learning rate of the critic network which is decreased by 0.05 every 5 time step until it reach $\eta_{c}(f)$ and stay thereafter; $N_{a}$, $N_{g}$ and $N_{c}$ are the internal cycle of the action network, goal network and critic network, respectively; $T_{a}, T_{g}$ and $T_{c}$ are the internal training error threshold for the action network, goal network and critic network, respectively.

\begin{tabular}{c||c|c|c|c|c|c}
\multicolumn{1}{l}{ Table 4: Specifical parameters of the GrADP based load frequency controller } \\
\hline \hline Parameters & $\eta_{a}(0)$ & $\eta_{g}(0)$ & $\eta_{c}(0)$ & $\eta_{a}(f)$ & $\eta_{g}(f)$ & $\eta_{g}(f)$ \\
\hline \begin{tabular}{c} 
value \\
\hline \hline Parameters
\end{tabular} & 0.3 & 0.3 & 0.3 & 0.05 & 0.05 & 0.05 \\
\hline value & $N_{a}$ & $N_{g}$ & $N_{c}$ & $T_{a}$ & $T_{g}$ & $T_{c}$ \\
\hline \hline
\end{tabular}

\section{Simulation Analysis}

In this section, comparisons of the PID controller, the PSO optimized fuzzy logic controller and the GrADP controller to damp the benchmark system frequency oscillation are presented. The parameters of the PID controller and the PSO optimized fuzzy logic controller are illustrated in Tab. 5. The convergence of the mean fitness value of PSO on 30 independent runs is illustrated in Fig. 7, where the fuzzy logic controller is optimized by PSO under system load disturbances between +0.3 p.u. to -0.3 p.u. with 0.05 interval.

We should notice that, there is only one PID controller to control the MT and the two EVs. We expect better control performance if there are three well coordinated PID controllers. However, the computation intensity and the cost of the subsystem for controllers will increase, and a coordination strategy 


\begin{tabular}{c||c||c||c}
\multicolumn{3}{c}{ Table 5: Parameters of the PID and PSO optimized fuzy logic controller } \\
\hline \hline Controllers & Parameters & Description & Value \\
\hline \hline \multirow{4}{*}{ PID } & $K_{p}$ & Proportional gain & 4 \\
\cline { 2 - 4 } & $K_{i}$ & Integral gain & 1.18 \\
\cline { 2 - 4 } & $K_{D}$ & Derivative gain & 0.5 \\
\hline \multirow{3}{*}{ Fuzzy Logic } & $K_{e}$ & Scaling factors 1 & $1.1743 \times 10^{3}$ \\
\cline { 2 - 4 } & $K_{e c}$ & Scaling factors 2 & 156.9976 \\
\cline { 2 - 4 } & $K_{u}$ & Scaling factors 3 & 0.0472 \\
\hline \hline
\end{tabular}

265 turbine are modeled as the power disturbance for the system. Moreover, the system damping performance with and without signal transmission delay are also considered in this simulation study.

\subsection{Case 1: Active Power Disturbance From PVs} the system. Specifically, a +0.05 p.u. step disturbance is applied at $5 \mathrm{~s}, \mathrm{a}-0.08$ p.u. step disturbance is applied at $50 \mathrm{~s}, \mathrm{a}+0.14 \mathrm{p} . \mathrm{u}$. step disturbance is applied at $100 \mathrm{~s}$, a -0.15 p.u. step disturbance is applied at $150 \mathrm{~s}$, a +0.16 p.u. step disturbance is applied at $200 \mathrm{~s}$, and a -0.20 p.u. step disturbance is applied at $250 \mathrm{~s}$. Under these sequential disturbances, the system frequency deviation using PID controller, PSO optimized fuzzy logic controller and GrADP controller with and without signal transmission delay is shown in Fig. 8. From Fig. 8(a), six learning and adapting stages of the GrADP controller can be observed, which correspond to the six sequential disturbances. Since the weights of the neural networks are randomly initialized in stage one ( $5 \mathrm{~s}$ to $50 \mathrm{~s}$ ), the GrADP controller does not generate proper control strategy. The performances of the original PID controller and the GrADP controller are similar. In stage two (50 s to $100 \mathrm{~s}$ ), GrADP approach utilizes the knowledge learned from stage one, which results in generating better control action. Thus improves the control performance 


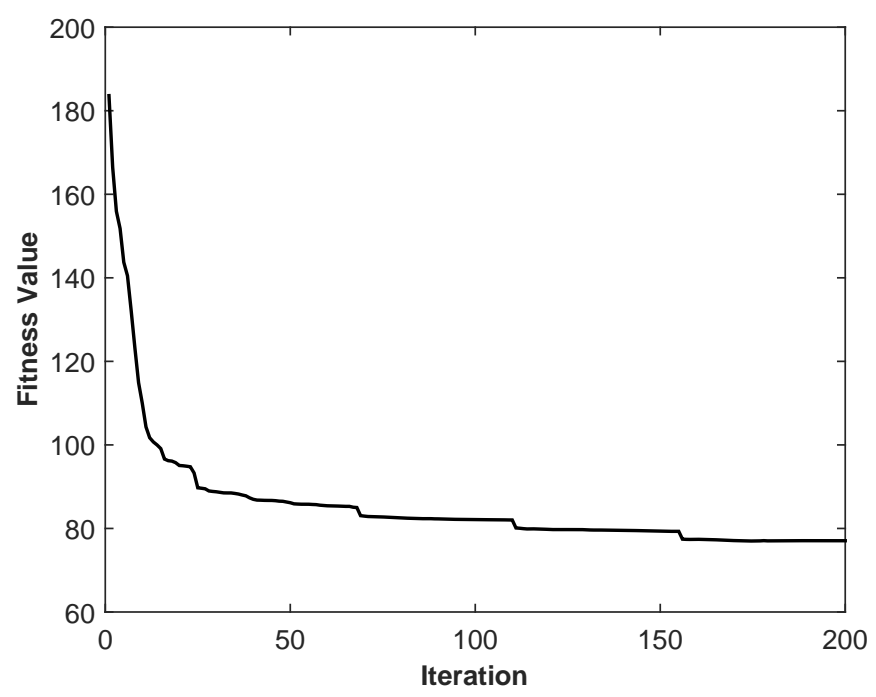

Figure 7: Mean fitness value on 30 independent runs to demonstrate the convergence of the PSO

compared with the original PID controller. In stage three to stage six (100 s to $300 \mathrm{~s}$ ), the control performance of the GrADP has been further improved. The peak values in the swings have been largely decreased and the system frequency oscillations have been quickly damped. While it can also be observed that the PSO optimized fuzzy logic controller performances quite well under all these disturbances, which represents that the PSO based method is effective. From Fig. 8(b), we could notice that the introduced signal transmission delay by the communication channel will degrade the damping performance of all the controllers, and the system frequency deviation will take longer time to become stable. It is also interesting to observe that the PSO optimized fuzzy logic controller is sensitive, where the performance has been largely affected by this delay.

The outputs of the MT, EVs using PID controller, PSO optimized fuzzy logic controller and GrADP controller with and without delay are shown in Fig. 9 respectively. Since the two EVs have identical system parameters and 


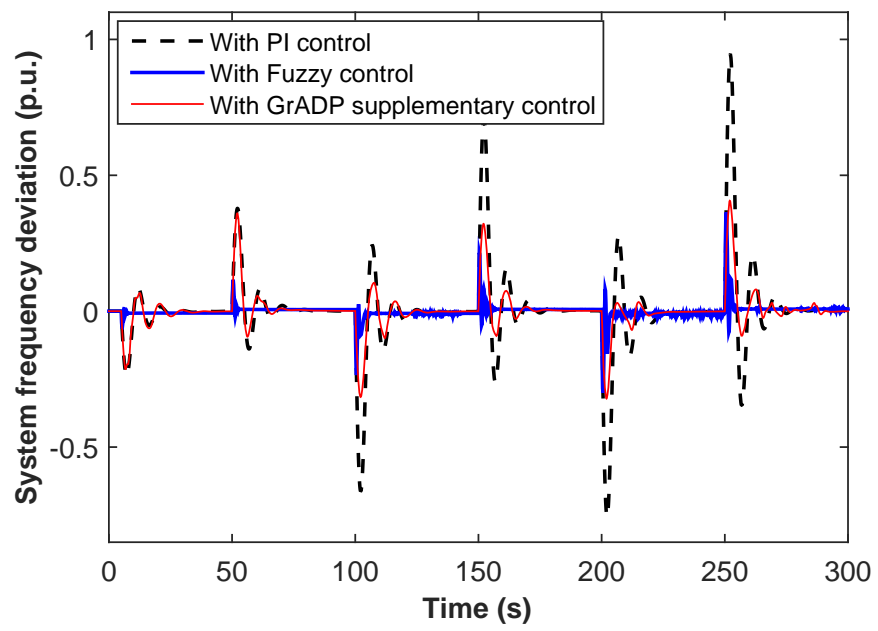

(a)

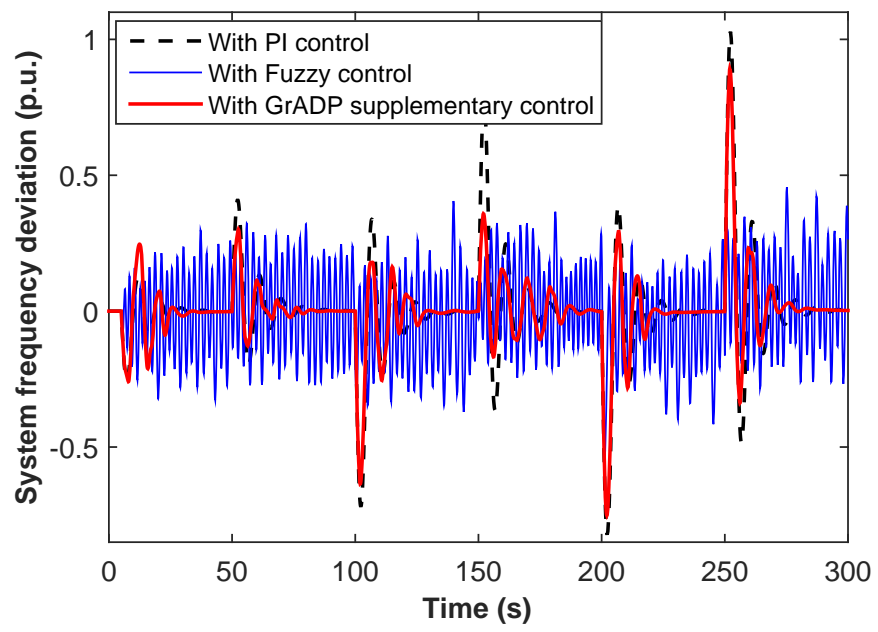

(b)

Figure 8: System frequency deviation with PID, fuzzy logic and GrADP controller in Case 1; (a) Without signal transmission delay; (b) With $200 \mathrm{~ms}$ signal transmission delay 
controllers, the outputs of the EVs are the same. Six stages of output response are clearly shown in this figure. Fig. 10 shows the corresponding GrADP output with and without delay in this case. As mentioned before, a limitation of \pm 0.02 is imposed to $\Delta u_{s}$. We can see that the GrADP controller adapts the control action according to different system disturbances, demonstrating the superior learning ability.

Fig. 11 shows the comparison of the $J_{I A E}$ in Case 1 with and without delay. Specifical, the controller performance under delay values of $100 \mathrm{~ms}, 200 \mathrm{~ms}, 300$ ms are all evaluated by $J_{I A E}$. It could be observed that the PSO optimized fuzzy logic controller shows the best control performance with $0 \mathrm{~ms}$ and $100 \mathrm{~ms}$ transmission delay. However, when the delay increase to $200 \mathrm{~ms}$ and $300 \mathrm{~ms}$, the performance has been largely degraded. Meanwhile, it could be observed that the adopted GrADP controller holds robust control performance even under 300 ms signal transmission delay.

\subsection{Case 2: Active Power Disturbance From Real Wind Fluctuation and PVs}

To investigate the feasibility of the LFC controllers in more complex and realistic situation, in Case 2, power fluctuation from real wind data plus power fluctuation from PVs are both applied to the system. As shown in Fig. 12 the real wind data from an offshore wind farm in Denmark 30] is adopted. Moreover, five sequential active power disturbances from PVs are also applied to the system. Specifical, a -0.05 p.u. step disturbance is applied at $5 \mathrm{~s}$, a +0.08 p.u. step disturbance is applied at $60 \mathrm{~s}, \mathrm{a}-0.14$ p.u. step disturbance is applied at $120 \mathrm{~s}$, a $+0.12 \mathrm{p} . \mathrm{u}$. step disturbance is applied at $180 \mathrm{~s}$, and a -0.15 p.u. step disturbance is applied at $240 \mathrm{~s}$. These two power fluctuations are added together as the total power disturbance to the smart grid, as shown in Fig. 13 .

Under these complex disturbances, the system frequency deviation using PID controller, PSO optimized fuzzy logic controller and GrADP controller with and without signal transmission delay is shown in Fig. 14, respectively. From Fig. 14(a), we could still observe five learning stages of the GrADP 

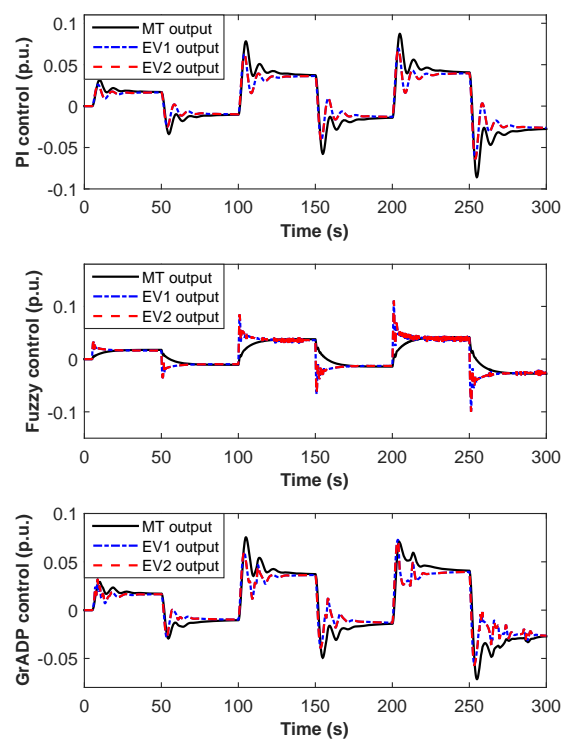

(a)
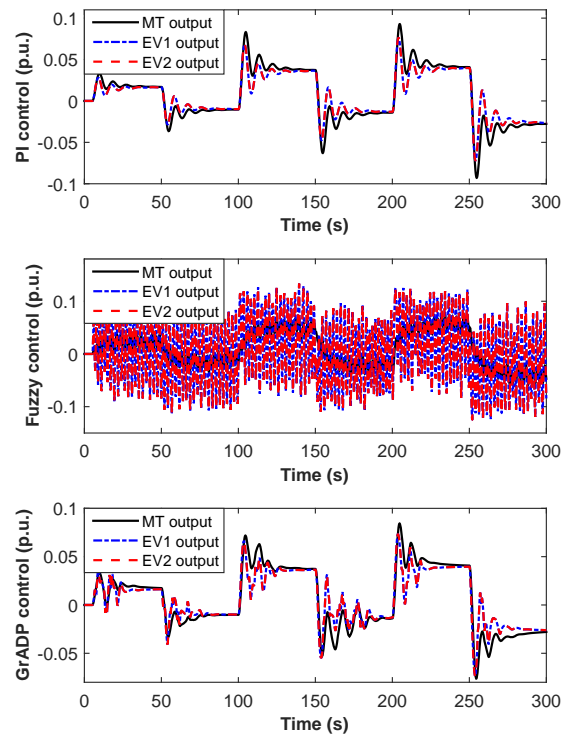

(b)

Figure 9: MT, EV 1 and EV 2 output with PID, fuzzy logic and GrADP controller in Case 1; (a) Without signal transmission delay; (b) With $200 \mathrm{~ms}$ signal transmission delay 


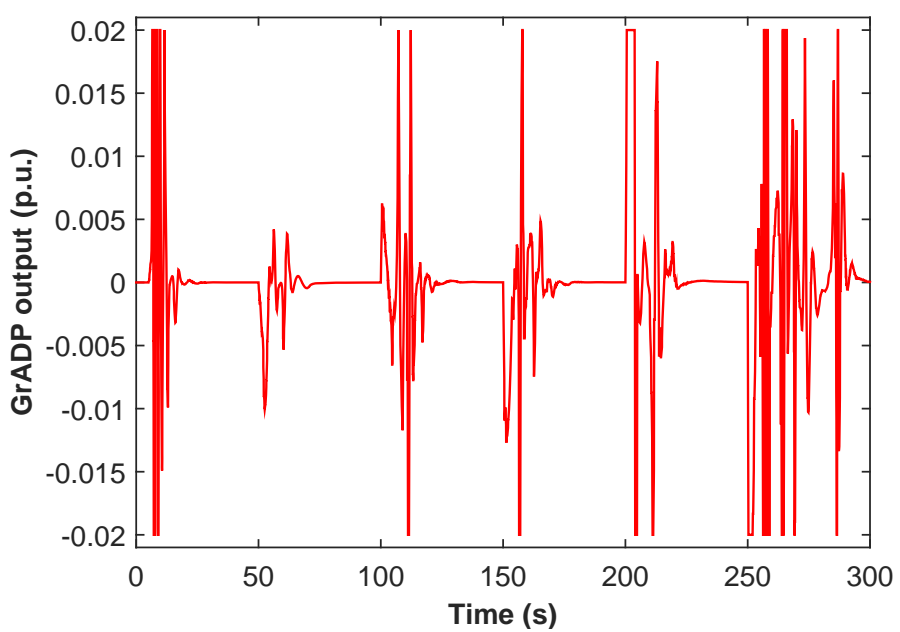

(a)

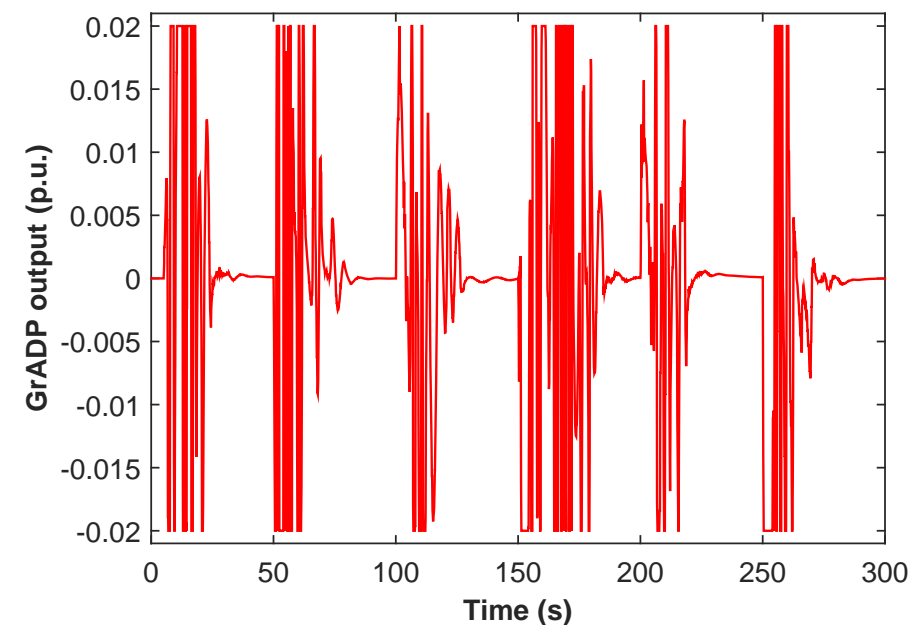

(b)

Figure 10: The corresponding GrADP output in Case 1; (a) Without signal transmission delay; (b) With $200 \mathrm{~ms}$ signal transmission delay 


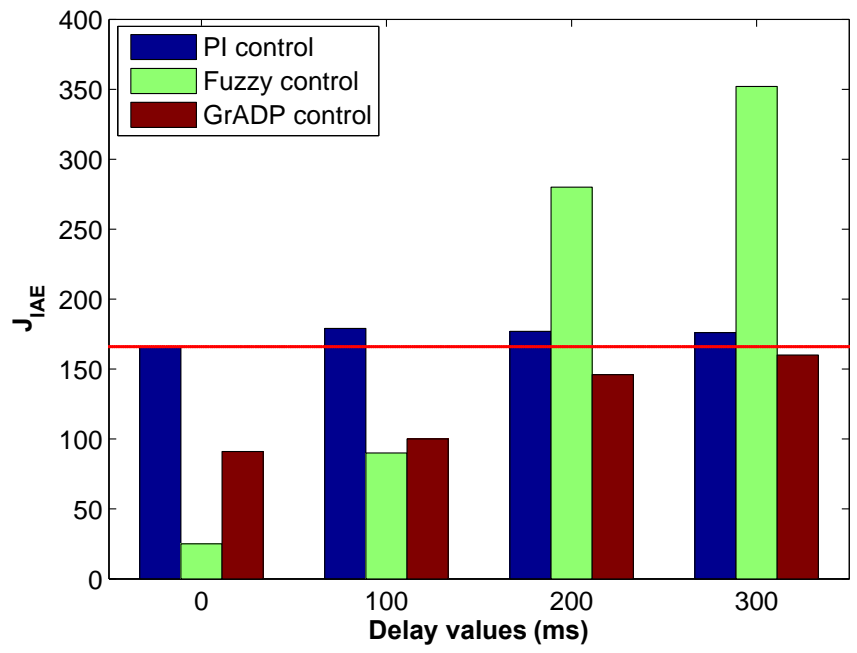

Figure 11: System performance of $J_{I A E}$ in Case 1

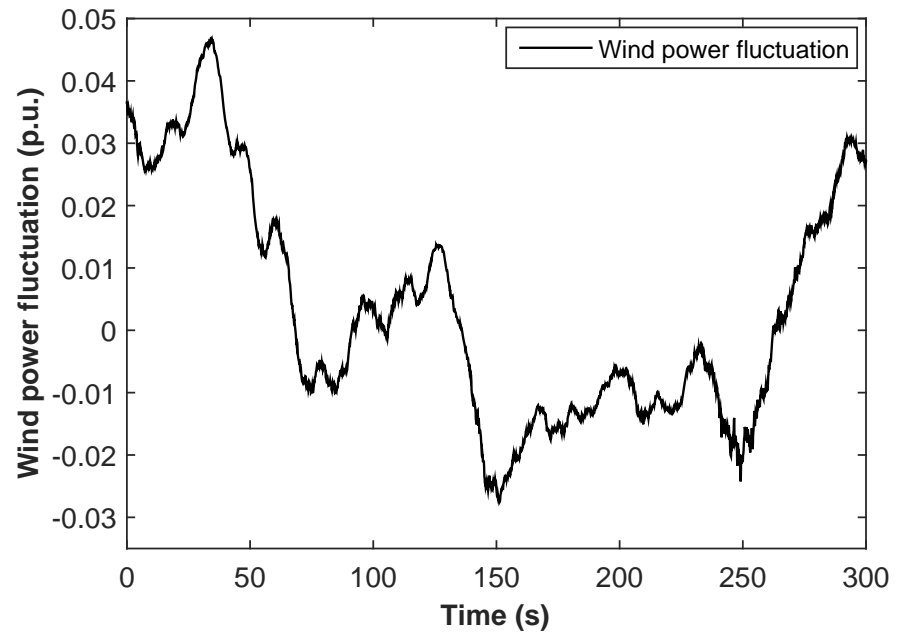

Figure 12: Power fluctuation from the wind turbine in Case 2 


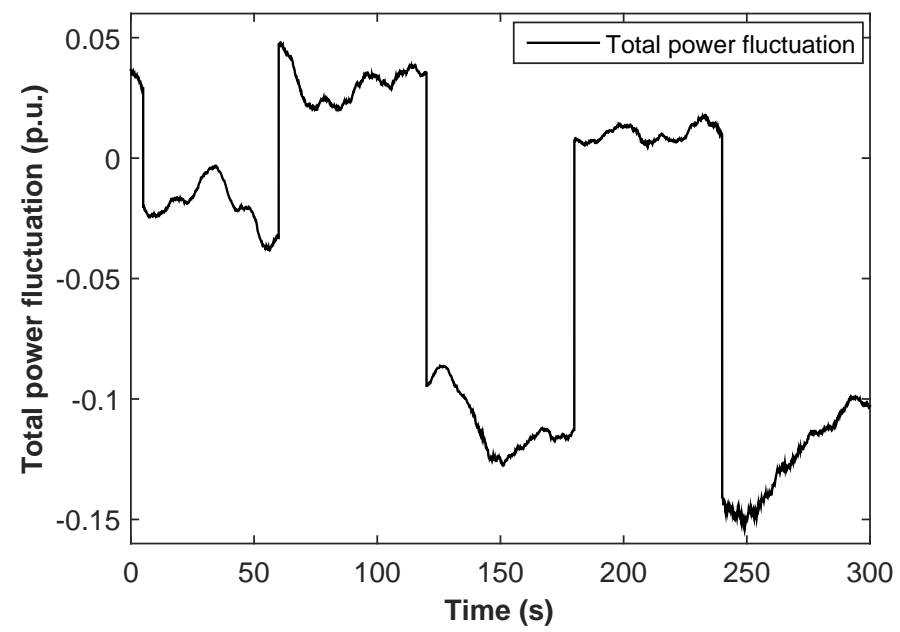

Figure 13: Total power disturbance from the wind turbine and the PVs in Case 2 


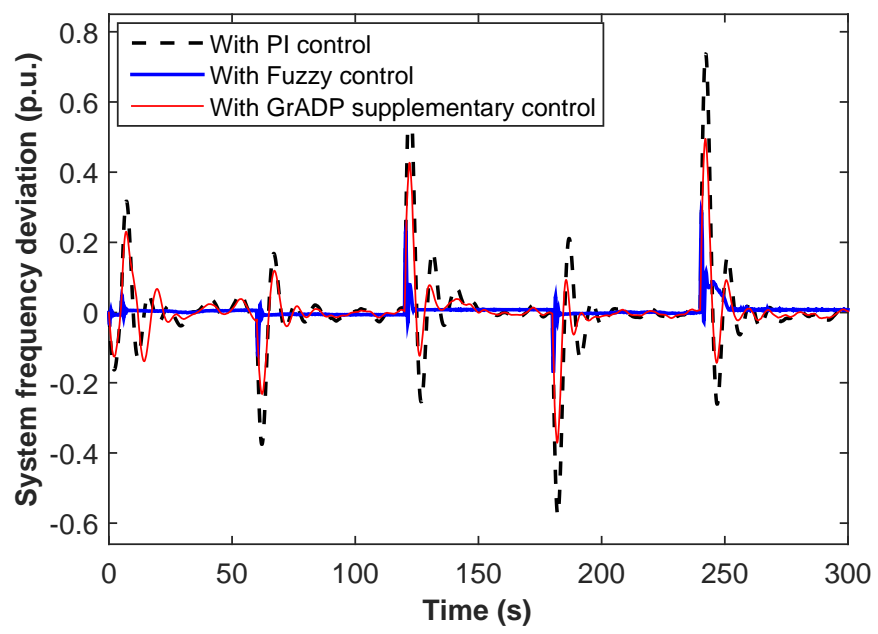

(a)

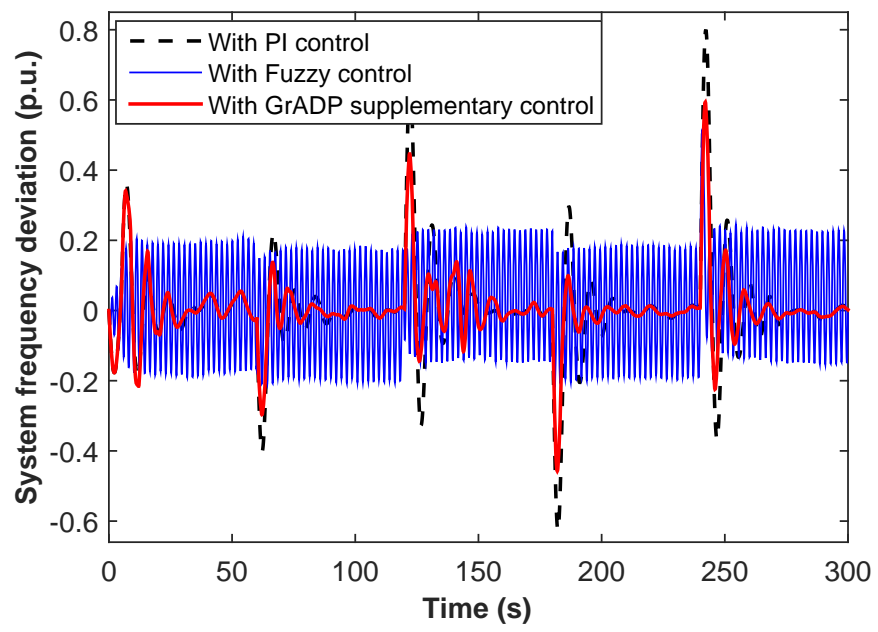

(b)

Figure 14: System frequency deviation with PID, fuzzy logic and GrADP controller in Case 2; (a) Without signal transmission delay; (b) With $200 \mathrm{~ms}$ signal transmission delay 


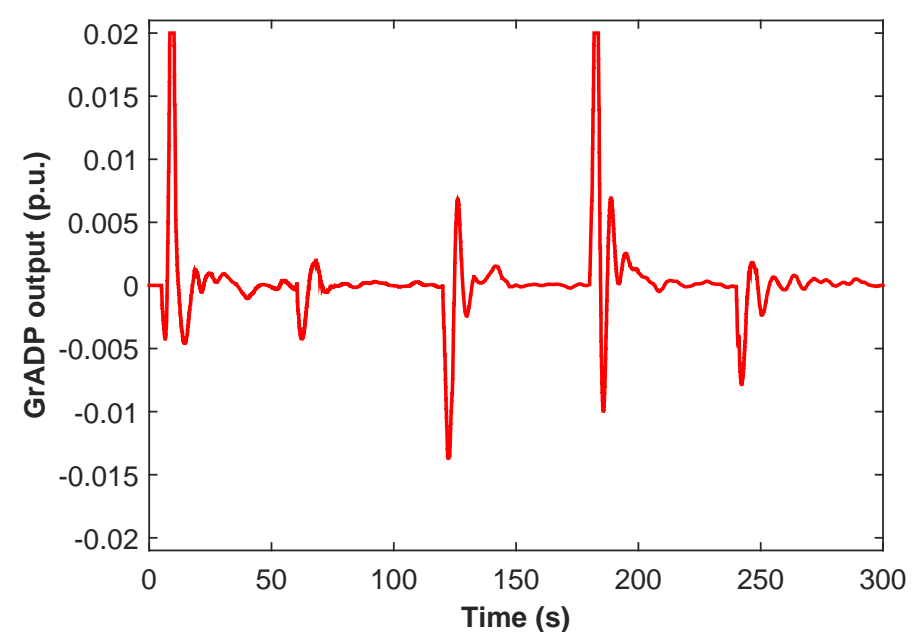

(a)

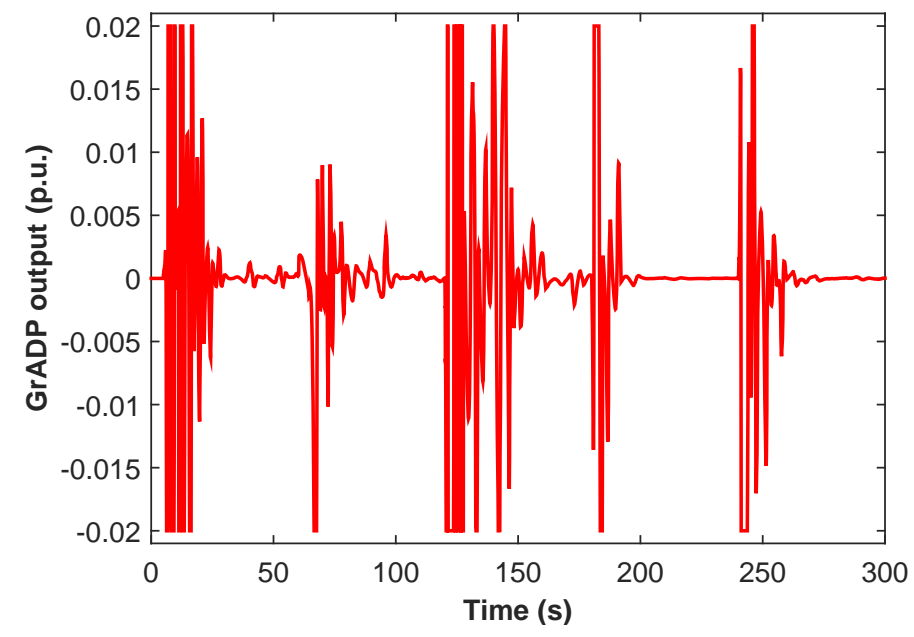

(b)

Figure 15: The corresponding GrADP output in Case 2; (a) Without signal transmission delay; (b) With $200 \mathrm{~ms}$ signal transmission delay 


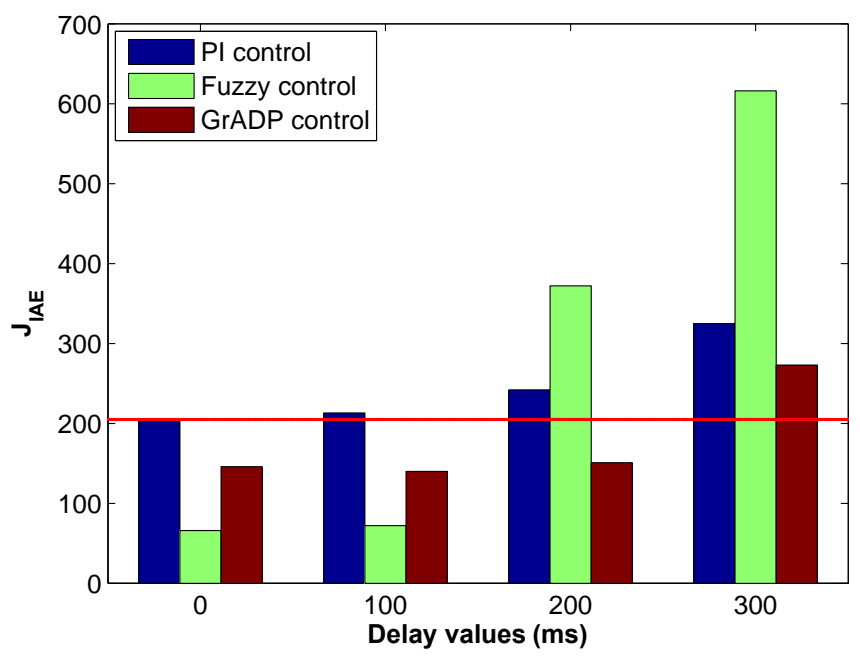

Figure 16: System performance of $J_{I A E}$ in Case 2

transmission delay.

\section{Conclusions}

This paper designed a supplementary controller to improve system frequency stability in island smart grid with EVs, PVs and wind turbine based on GrADP. The controller design, including the input signal selection and the reinforcement signal design, were introduced in details. Comparative studies of the original PID controller and the PSO optimized fuzzy logic controller with signal transmission delay were carried out through two study cases. Under several sequential active power disturbances and real wind power fluctuation, the simulation results demonstrated the superior instant learning ability and robust control effect of the designed GrADP based load frequency controller.

Optimal intelligent control methods have been introduced into power system operation for more than ten years. However, considerable efforts are still needed for real power engineering applications. This paper may shed some light on narrowing the gap between the theoretic research and realistic applications. In the future work, more detailed EV model and larger benchmark power system are 
required to test the intelligent control method. Considering the adaptive ability of damping system oscillation of the GrADP controller, it may be interesting to apply it to other power system oscillation problems, such as low-frequency oscillation problems in connected grids by long distance tie-lines. Moreover, effective methods to address signal transmission delay in the future open communication networks are also needed. This issue could be addressed by developing more advanced delay-dependent controller as well as calculating the delay margin for engineering instruction applications in the future studies.

\section{References}

[1] Y. Tang, J. Yang, J. Yan, Z. Zeng, H. He, Frequency control using online learning method for island smart grid with EVs and PVs, in: Neural Networks (IJCNN), 2014 International Joint Conference on, 2014, pp. 1440-1446. doi:10.1109/I JCNN . 2014.6889829.

[2] J. A. P. Lopes, F. J. Soares, P. M. R. Almeida, Integration of electric vehicles in the electric power system, Proceedings of the IEEE 99 (1) (2011) $168-183$.

[3] P. Kundur, Power System Stability and Control, Mc Graw-Hill, New York, USA, 1994.

[4] H. Liu, Z. Hu, Y. Song, J. Lin, Decentralized vehicle-to-grid control for primary frequency regulation considering charging demands, Power Systems, IEEE Transactions on 28 (3) (2013) 3480-3489. doi:10.1109/TPWRS. 2013.2252029

[5] K. Shimizu, T. Masuta, Y. Ota, A. Yokoyama, Load frequency control in power system using vehicle-to-grid system considering the customer convenience of electric vehicles, in: Power System Technology (POWERCON), 2010 International Conference on, IEEE, 2010, pp. 1-8. 
[6] J. R. Pillai, B. Bak-Jensen, Integration of vehicle-to-grid in the western Danish power system, Sustainable Energy, IEEE Transactions on 2 (1) (2011) 12-19.

[7] M. Singh, P. Kumar, I. Kar, Implementation of vehicle to grid infrastructure using fuzzy logic controller, Smart Grid, IEEE Transactions on 3 (1) (2012) 565-577.

[8] M. Datta, T. Senjyu, Fuzzy control of distributed PV inverters/energy storage systems/electric vehicles for frequency regulation in a large power system, Smart Grid, IEEE Transactions on 4 (1) (2013) 479-488. doi: 10.1109/TSG.2012.2237044

[9] T. Masuta, A. Yokoyama, Supplementary load frequency control by use of a number of both electric vehicles and heat pump water heaters, Smart Grid, IEEE Transactions on 3 (3) (2012) 1253-1262.

[10] S. Vachirasricirikul, I. Ngamroo, Robust LFC in a smart grid with wind power penetration by coordinated V2G control and frequency controller,

1 Smart Grid, IEEE Transactions on 5 (1) (2014) 371-380. doi:10.1109/ TSG.2013.2264921.

[11] M. Toge, Y. Kurita, S. Iwamoto, Supplementary load frequency control with storage battery operation considering SOC under large-scale wind power penetration, in: Power and Energy Society General Meeting (PES), 2013 IEEE, 2013, pp. 1-5. doi:10.1109/PESMG.2013.6672323.

[12] W. Guo, F. Liu, S. Mei, J. Si, D. He, R. Harley, Approximate dynamic programming based supplementary frequency control of thermal generators in power systems with large-scale renewable generation integration, in: PES General Meeting - Conference Exposition, 2014 IEEE, 2014, pp. 1-5. doi: 10.1109/PESGM. 2014.6939104.

415 [13] J. Si, Y.-T. Wang, Online learning control by association and reinforcement, 
Neural Networks, IEEE Transactions on 12 (2) (2001) 264 -276. doi: $10.1109 / 72.914523$

[14] X. Fang, H. He, Z. Ni, Y. Tang, Learning and control in virtual reality for machine intelligence, in: Intelligent Control and Information Processing (ICICIP), 2012 Third International Conference on, IEEE, 2012, pp. 63-67.

[15] Z. Ni, H. He, J. Wen, Adaptive learning in tracking control based on the dual critic network design, Neural Networks and Learning Systems, IEEE Transactions on 24 (6) (2013) 913-928. doi:10.1109/TNNLS.2013. 2247627.

[16] Z. Ni, H. He, J. Wen, X. Xu, Goal representation heuristic dynamic programming on maze navigation, Neural Networks and Learning Systems, IEEE Transactions on 24 (12) (2013) 2038-2050. doi:10.1109/TNNLS. 2013.2271454

[17] X. Sui, Y. Tang, H. He, J. Wen, Energy-storage-based low-frequency oscillation damping control using particle swarm optimization and heuristic dynamic programming, Power Systems, IEEE Transactions on 29 (5) (2014) 2539-2548. doi:10.1109/TPWRS.2014.2305977.

[18] Y. Tang, H. He, J. Wen, J. Liu, Power system stability control for a wind farm based on adaptive dynamic programming, Smart Grid, IEEE Transactions on 6 (1) (2015) 166-177. doi:10.1109/TSG.2014.2346740.

[19] J. Pahasa, I. Ngamroo, Coordinated control of wind turbine blade pitch angle and PHEVs using MPCs for load frequency control of microgrid,

п Systems Journal, IEEE PP (99) (2014) 1-9. doi:10.1109/JSYST. 2014. 2313810

[20] H. Wu, K. S. Tsakalis, G. T. Heydt, Evaluation of time delay effects to widearea power system stabilizer design, Power Systems, IEEE Transactions on 19 (4) (2004) 1935-1941. 
[21] H. He, Self-Adaptive Systems for Machine Intelligence, Wiley, USA, 2011.

[22] H. He, Z. Ni, J. Fu, A three-network architecture for on-line learning and optimization based on adaptive dynamic programming, Neurocomputing 78 (1) (2012) 3-13.

[23] H. Shayeghi, A. Jalili, H. Shayanfar, Multi-stage fuzzy load frequency control using PSO, Energy Conversion and Management 49 (10) (2008) 25702580.

[24] H. Bevrani, P. Daneshmand, Fuzzy logic-based load-frequency control concerning high penetration of wind turbines, Systems Journal, IEEE 6 (1) (2012) 173-180. doi:10.1109/JSYST.2011.2163028.

[25] H. Bevrani, F. Habibi, P. Babahajyani, M. Watanabe, Y. Mitani, Intelligent frequency control in an AC microgrid: Online PSO-based fuzzy tuning approach, Smart Grid, IEEE Transactions on 3 (4) (2012) 1935-1944. doi: 10.1109/TSG.2012.2196806

[26] A. Bartoszewicz, A. Nowacka-Leverton, ITAE optimal sliding modes for third-order systems with input signal and state constraints, Automatic Control, IEEE Transactions on 55 (8) (2010) 1928-1932. doi:10.1109/ TAC.2010.2049688,

[27] Y. Tang, H. He, J. Wen, Comparative study between HDP and PSS on DFIG damping control, in: Computational Intelligence Applications In

1 Smart Grid (CIASG), 2013 IEEE Symposium on, 2013, pp. 59-65. doi: 10.1109/CIASG.2013.6611499.

[28] Y. Tang, H. He, J. Wen, Adaptive control for an HVDC transmission link with FACTS and a wind farm, in: in Proc. IEEE Innovative Smart Grid Technologies Conference (ISGT'13), Washington, February, 2013.

[29] Y. Tang, H. He, Z. Ni, J. Wen, X. Sui, Reactive power control of gridconnected wind farm based on adaptive dynamic programming, Neurocomputing 125 (0) (2014) 125-133. 
[30] Wind data report, Tech. rep., Technical University of Denmark (March 2014).

URL http://www.winddata.com/ 